\title{
Electron impact dissociation cross sections for $\mathbf{C}_{2} \mathbf{F}_{6}$
}

\author{
D W Flaherty ${ }^{1}$, M A Kasper ${ }^{1}$, J E Baio ${ }^{1}$, D B Graves ${ }^{1}$, \\ H F Winters ${ }^{1,3}$, C Winstead, ${ }^{2}$ and V McKoy ${ }^{2}$ \\ ${ }^{1}$ Department of Chemical Engineering, University of California, Berkeley, CA 94720, USA \\ ${ }^{2}$ A. A. Noyes Laboratory of Chemical Physics, California Institute of Technology, Pasadena, \\ CA 91125, USA \\ E-mail: Htxc88a@yahoo.com (H F Winters)
}

Received 5 August 2006, in final form 29 August 2006

Published 29 September 2006

Online at stacks.iop.org/JPhysD/39/4393

\begin{abstract}
Absolute total dissociation cross sections for electron impact, $\sigma_{\mathrm{t}, \mathrm{diss}}$, from 8 to $700 \mathrm{eV}$ are reported for $\mathrm{C}_{2} \mathrm{~F}_{6}$. A dense set of data was obtained in the technologically important $8-30 \mathrm{eV}$ energy range relevant to modelling the type of plasmas used in both fundamental and applied scientific investigations. The threshold for dissociation was found to be $12.0 \pm 0.2 \mathrm{eV}$ and appears to be associated with a Rydberg state. Estimated values for the total neutral dissociation cross section, $\sigma_{\text {neut,diss }}$, were obtained by subtracting the ionization cross sections (all ionizing events cause dissociation) from the total dissociation cross section. It is shown that a calibration error in a paper by one of us (HFW) caused a distortion of several previous investigations. When the data from the present work are used to recalibrate data from swarm experiments, agreement becomes quite reasonable. There is now a consistent set of data obtained from several investigators which describe the dissociation of $\mathrm{C}_{2} \mathrm{~F}_{6}$. Neutral dissociation cross sections are obtained from electron-impact excitation calculations and found to be in reasonable agreement with measurements over most of the energy range.
\end{abstract}

\section{Introduction}

Electron impact dissociation of molecules into fragments is one of the most important processes in molecular gas discharges. Indeed, the process that makes most technologies based on such plasmas operative is electron impact dissociation of inert feed gases to generate chemically reactive species such as fluorine atoms, nitrogen atoms, $\mathrm{CF}_{2}$ radicals. These discharges are employed in numerous steps in microelectronics fabrication including plasma etching, plasma-enhanced chemical vapour deposition, photo resist stripping and surface cleaning and are also widely used in other industries.

The accuracy and reliability of plasma chemistry models hinge on the availability of basic data describing electronmolecule collisions, especially at energies near the dissociation thresholds, which are typically of the order of $10 \mathrm{eV}$.

3 Author to whom any correspondence should be addressed.
The majority of electrons in these plasmas generally have energies below $30 \mathrm{eV}$; therefore, electron-molecule collisions at energies less than about $30 \mathrm{eV}$ are highly relevant to modelling. Electron-molecule collision data provide the rate at which reactive species are created and thus place an upper limit on many processes.

Among the fluorocarbons widely used as precursor gases in plasma processing, a complete set of electron collision data is only available for $\mathrm{CF}_{4}$ [1]. Reliable cross section data for total dissociation, $\sigma_{\mathrm{t}, \text { diss }}$, total ionization, $\sigma_{\mathrm{t}, \mathrm{ion}}$, and neutral dissociation, $\sigma_{\text {neut,diss }}$ of these gases are critical to plasma process modelling and process design. For example, the problems associated with emission of these stable greenhouse gases into the environment have prompted studies of plasmabased dissociation and destruction of perfluorocarbon (PFC) and hydrofluorocarbon (HFC) gases [2].

There have been numerous measurements of $\sigma_{\mathrm{t}, \text { ion }}$ for $\mathrm{C}_{2} \mathrm{~F}_{6}$ from the threshold to several hundred eV; [3] however, total dissociation cross sections have been measured only at 
$22 \mathrm{eV}$ and at a few selected energies above $70 \mathrm{eV}$. We are not aware of any published absolute values of $\sigma_{\mathrm{t} \text {,diss }}$ for $\mathrm{C}_{2} \mathrm{~F}_{6}$ in the critical near-threshold region. Moreover, measurements of $\sigma_{\text {neut,diss }}$ for many gases are scarce due to difficulties in detecting neutral fragments, and the authors are unaware of any published data for $\sigma_{\text {neut,diss }}$ of $\mathrm{C}_{2} \mathrm{~F}_{6}$ derived from electron beam experiments. Measurements of cross sections for dissociation of a few prototypical gases into neutral fragments are furthermore essential for assessing the calculated values of these cross sections. With such validation, calculated cross sections, particularly near the threshold, may be used with greater confidence where experimental data are unavailable.

In the present work, the method of Winters et al [4-8] has been used to measure $\sigma_{\mathrm{t} \text {,diss }}$ for $\mathrm{C}_{2} \mathrm{~F}_{6}$ at closely-spaced energies from 8 to $30 \mathrm{eV}$ and at selected higher energies up to $700 \mathrm{eV}$. These studies show that previous measurements overestimated $\sigma_{\mathrm{t} \text {,diss }}$, especially at energies near the dissociation threshold, where the values recommended here are approximately twothirds of the previous measurements (probably because of uncertainty about the electron energy in a region of rapidly changing cross section). Values for $\sigma_{\text {neut,diss }}$ are obtained by subtracting published values of $\sigma_{\mathrm{t} \text {,ion }}$ from $\sigma_{\mathrm{t} \text {,diss }}$. This procedure has previously provided values of these cross sections for $\mathrm{CF}_{4}$ in agreement with independent measurements by $\mathrm{Mi}$ and Bonham [9]. In addition, cross sections for $\sigma_{\text {neut,diss }}$ of $\mathrm{C}_{2} \mathrm{~F}_{6}$ near the threshold have been calculated employing the Schwinger multichannel method [10]. It is shown that these calculated cross sections agree quite well with those derived from the experiment.

\section{Method}

The method and analysis used to measure the total dissociation cross section have been described in detail [4] and are only summarized here. A monoenergetic electron beam of known energy and current is passed through a sealed tube containing the target gas at room temperature and a predetermined initial pressure. If all neutral and ionic fragments created during the passage of the beam through the target gas are adsorbed at the walls, the rate of change in the total gas pressure can be related to the total (neutral plus ionic) dissociation cross section. The ideal gas law accurately describes the behaviour of the gas at the experimental pressure $\left(\sim 5 \times 10^{-5}\right.$ Torr), and the number of fragmentation events can thus be related to the pressure change. The gas molecules do not react with one another and all fragmentation events can be attributed either to interactions with the tungsten filament or to electronmolecule collisions. By just changing the electron beam from sub-threshold energies to a determined experimental energy, it is possible to discern the amount of dissociation initiated by electron impact.

Relating the change in pressure to the number of fragmentation events through the ideal gas law implies several assumptions about the behaviour of both the parent gas and the dissociation products within the apparatus. Three criteria established by Winters and Inokuti [4] must be satisfied to ensure accurate cross section measurements. First, undissociated ions and undissociated excited neutrals cannot leave the gas phase as a result of surface reactions. Second, all products of dissociation must irreversibly adsorb to the

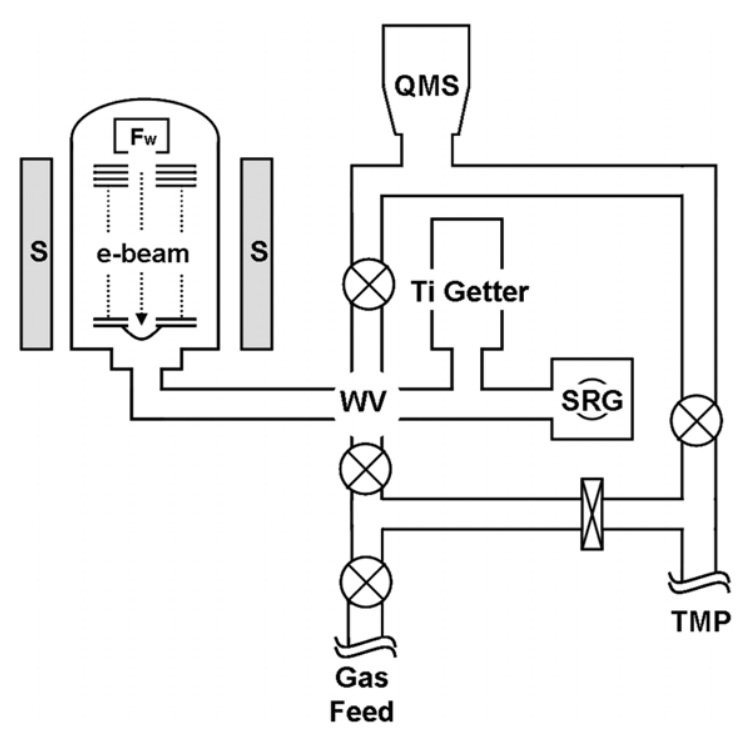

Figure 1. Schematic of experimental apparatus.

chamber walls or getter. Third, fragments must not recombine on surfaces within the apparatus to reform the parent gas. For $\mathrm{C}_{2} \mathrm{~F}_{6}$, mass spectrometry and electron-impact energy loss spectra indicate that all electronic excitation and ionization lead to impulsive dissociation of the parent molecule [11, 12], and the use of a titanium getter prevents surface recombination of fragments and eliminates desorption of impurity gases as a factor.

\section{Apparatus description}

\subsection{Overview}

All measurements of the absolute $\sigma_{\mathrm{t} \text {,diss }}$ are made within a closed working volume (WV), containing an (MKS) spinning rotor gauge (SRG), a titanium getter and 'the tube,' which generates and controls the electron beam responsible for dissociating the parent gas (cf figure 1). The WV is linked via an ultra-high vacuum valve to a second volume containing an Inficon mass spectrometer, which can be pumped independently of the WV. A solenoid surrounding the experimental tube generates a magnetic field to collimate the electron beam. After high temperature bake-out and long pumping time, the WV of the system could be sealed and maintained at a base pressure below the sensitivity of the SRG $\left(<1 \times 10^{-8}\right.$ Torr $)$ for several hours.

\subsection{The tube}

The experimental tube is the same as that used by Winters and Inokuti and is described by them in detail $[4,5]$. Figures 1 and 2 are schematics of the apparatus. Electrons are emitted from a 0.01 inch diameter tungsten filament, $F_{W}$, located at the top of the tube and collected by an electron sink, Target, at the opposite end. The $250 \mathrm{G}$ solenoid, S, surrounding the bell jar containing the tube aids in forming the electron beam, whose energy is controlled by the voltages on the lettered electrodes along the length of the tube (cf figure 2). Simultaneous monitoring of all electrodes throughout the experimental tube 


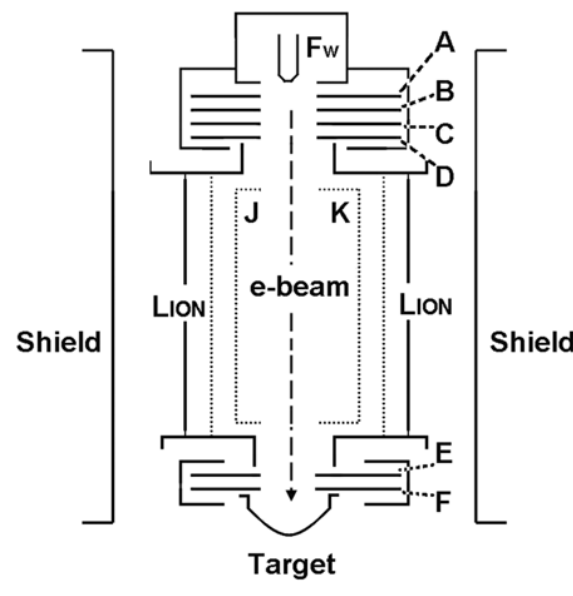

Figure 2. Diagram of the experimental tube.

established that the number of secondary electrons, originating from the sink electrode and also contributing to dissociation, was greater than thought in previous studies and, in fact, the previous values of $\sigma_{\mathrm{t} \text {, diss }}$ appeared larger than they actually were. A $300 \mathrm{~V}$ bias between electrode $\mathrm{F}$ and target was found to suppress most secondary electrons, alleviating the problem. Some dissociation does occur between electrode $\mathrm{F}$ and Target due to the $300 \mathrm{~V}$ bias. However, this bias is always present, so dissociation in this region does not affect the cross section measurements.

For measurements of $\sigma_{\mathrm{t}, \text { ion }}$, a small electric field perpendicular to the electron beam is used to draw out ions without affecting the energy of the electron beam. The electric field is created by placing potentials of +2 and $-2 \mathrm{~V}$ on the two semi-circular tungsten grids, $\mathrm{J}$ and $\mathrm{K}$, while the ion current is measured from the cylindrical electrode 'Shield', surrounding the dissociation path. A set of small cylinders, $\mathrm{L}_{\mathrm{ION}}$, is used to set the path length from which the ions are extracted.

\section{Experimental}

\subsection{Procedure}

Each experiment begins by filling the $\mathrm{WV}$, with the parent gas, $\mathrm{C}_{2} \mathrm{~F}_{6}$, to a pressure of $5 \times 10^{-5}$ Torr and sealing the system (cf figure 1). The experiment then proceeds in three distinct phases, during each of which the pressure within the closed volume is monitored using the SRG, while the emission current and the temperature of the tungsten filament are held constant. In the first and third phases, the electron beam is kept at subthreshold energies (except for the region between electrode $\mathrm{F}$ and target) to determine a background fragmentation rate. In the second phase, the electron beam energy is set to the desired value to determine the total fragmentation rate due to energetic electron-molecule collisions. Values for $\sigma_{\mathrm{t} \text {,diss }}$ are obtained by comparing the slopes of the logarithm of pressure versus time for the corresponding experimental phases. All slopes are derived using least-squares fits, typically resulting in correlation to at least $R^{2}=0.99$. As discussed, secondary electrons originating from the sink electrode can cause a significant amount of dissociation and produce uncertainty in the current reaching the electron sink. Increasing the target bias to 300 from $75 \mathrm{~V}$ resulted in approximately an $8 \%$ decrease in the apparent value of $\sigma_{\mathrm{t}, \text { diss }}$ at electron energies greater than $70 \mathrm{eV}$.

\subsection{Calibration technique}

A small but finite voltage drop across the filament and space charge effects due to the electrons cause some initial uncertainty in the energy of the electron beam, and it is therefore necessary to calibrate the energy scale of the apparatus. To this end, the total ionization cross section of argon was measured from 13 to $300 \mathrm{eV}$. The ionization threshold was determined by extrapolation of a linear fit to the near-threshold data. The difference between the published threshold for ionization $(15.76 \mathrm{eV})$ and the threshold so determined $(14.67 \mathrm{eV})$ was used to correct the electron beam energy scale of all experiments. (The original calibration of this type by Winters and Inokuti seems to have been slightly flawed, leading to a larger uncertainty in their $22 \mathrm{eV}$ data point than in those taken at higher energy.)

The most accurate cross sections were obtained with the electron sink biased $+300 \mathrm{~V}$ relative to electrodes $\mathrm{E}$ and $\mathrm{F}$. Measurements of $\sigma_{\mathrm{t}, \text { ion }}$ for argon provide not only a correction for the energy scale but also the ion collection efficiency of the system, which was found to be $71 \%$. With this correction, our measured $\sigma_{\mathrm{t}, \text { ion }}$ for argon agrees closely with accepted values published by Rapp and Englander-Golden [13] from threshold to $300 \mathrm{eV}$.

In many ways, measurement of $\sigma_{\mathrm{t} \text {,diss }}$ is simpler and probably more accurate than measurement of $\sigma_{\mathrm{t}, \text { ion }}$. This is particularly true for $\mathrm{C}_{2} \mathrm{~F}_{6}$, where all excitations lead to dissociation. Dissociation measurements are not susceptible to error from uncertainty in the ion collection path length or to double counting due to ion-induced secondary electrons or to the presence of doubly charged ions. Because all electronic excitations of $\mathrm{C}_{2} \mathrm{~F}_{6}$ lead to dissociation, the total ionization cross section should never exceed the total dissociation cross section. The $\mathrm{C}_{2} \mathrm{~F}_{6}$ ionization cross section measured by Basner et al is everywhere smaller than our $\sigma_{\mathrm{t}, \text { diss }}$, and we consider it to be quite reliable. [11]

\subsection{Error analysis}

There are at least four separate sources of experimental error. The path length of the electron beam constitutes the single largest source of error, estimated to be $\pm 9 \%$. The path length uncertainty is caused by our inability to track individual electrons from source to destination. Random error from fitting the pressure versus time data contributes approximately $\pm 6 \%$, while errors in the electron beam current and volume measurements contribute $\pm 3 \%$ and $\pm 2 \%$, respectively. Combining these errors in standard fashion [14] leads to an estimate of $\pm 11 \%$ for the overall error in the measured values of $\sigma_{\mathrm{t} \text {,diss }}$.

Ionization, metastable excitation, excitation to allowed states and dissociation-all can be measured by alternative methods, but these methods require a measurement of the absolute value of the gas density. It is much easier to determine the slope of the pressure versus the time curve than it is to determine the absolute pressure. Also, the present dissociation experiments have an inherent collection efficiency of one if the criteria described in section 2 are rigorously applied, because 
the number of molecules leaving the gas phase is determined with a high degree of accuracy by the change in pressure. For example, there is no possibility of double-counting because of ion-induced secondary electrons or doubly charged ions. Additionally, the path length over which the excitation occurs is well defined by the geometrical path length.

\section{Computational}

An estimate of $\sigma_{\text {neut,diss }}$ near threshold can be obtained from $a b$ initio calculations of electron-impact excitation cross sections under the assumptions that all electronically excited states of $\mathrm{C}_{2} \mathrm{~F}_{6}$ are dissociative and that they produce only neutral fragments (no dipolar dissociation). We used a parallel implementation [15] of the Schwinger multichannel method $[10,16]$ to compute electron-impact excitation cross sections for eight low-lying electronic states of $\mathrm{C}_{2} \mathrm{~F}_{6}: \quad\left(6 \mathrm{a}_{1 \mathrm{~g}} \rightarrow\right.$ $\left.7 \mathrm{a}_{1 \mathrm{~g}}\right)^{1,3} \mathrm{~A}_{1 \mathrm{~g}},\left(6 \mathrm{a}_{1 \mathrm{~g}} \rightarrow 6 \mathrm{e}_{\mathrm{u}}\right)^{1,3} \mathrm{E}_{\mathrm{u}},\left(6 \mathrm{a}_{1 \mathrm{~g}} \rightarrow 6 \mathrm{a}_{2 \mathrm{u}}\right)^{1,3} \mathrm{~A}_{2 \mathrm{u}}$, and $\left(6 \mathrm{a}_{1 \mathrm{~g}} \rightarrow 7 \mathrm{e}_{\mathrm{u}}\right)^{1,3} \mathrm{E}_{\mathrm{u}}$. The calculations employed the 6$311+\mathrm{G}(d)$ basis set [17] for description of both bound and unbound electrons. Standard diffuse and d orbital exponents were used, namely 0.0438 (sp) and 0.626 (d) for C and 0.1076 (sp) and 1.75 (d) for F, and all six Cartesian components of the $d$ Gaussians were retained in the calculation. All cross sections were computed at the $D_{3 \mathrm{~d}}$ ground-state equilibrium nuclear geometry as determined by second-order MöllerPlesset perturbation theory, namely $r_{\mathrm{CC}}=1.5275 \AA, r_{\mathrm{CF}}=$ $1.3386 \AA$ and $L_{\mathrm{CCF}}=109.6825^{\circ}$. In the cross-section calculations, we used a restricted Hartree-Fock description of the ground state, while excited states were described within the improved virtual-orbital (IVO) approximation [18]. The calculations were carried out in a few-channel approximation, in which the singlet and triplet states corresponding to the same configuration were coupled only to each other and to the ground state. The scattering programme requires that all states having the same nominal configuration have the same spatial wavefunction; we therefore used the triplet IVOs to form both triplet and singlet excited states. Thresholds computed in this manner are 13.52 and $15.00 \mathrm{eV},\left(6 \mathrm{a}_{1 \mathrm{~g}} \rightarrow 6 \mathrm{e}_{\mathrm{u}}\right)$ ${ }^{1,3} \mathrm{E}_{\mathrm{u}} ; 14.29$ and $14.40 \mathrm{eV},\left(6 \mathrm{a}_{1 \mathrm{~g}} \rightarrow 7 \mathrm{a}_{1 \mathrm{~g}}\right){ }^{1,3} \mathrm{~A}_{1 \mathrm{~g}} ; 15.10$ and $15.22 \mathrm{eV},\left(6 \mathrm{a}_{1 \mathrm{~g}} \rightarrow 6 \mathrm{a}_{2 \mathrm{u}}\right){ }^{1,3} \mathrm{~A}_{2 \mathrm{u}}$; and 15.61 and $15.86 \mathrm{eV}$, $\left(6 \mathrm{a}_{1 \mathrm{~g}} \rightarrow 7 \mathrm{e}_{\mathrm{u}}\right)^{1,3} \mathrm{E}_{\mathrm{u}}$, where in each case the triplet energy is listed before the singlet energy. IVO thresholds typically are higher than experimental vertical thresholds by 1 or $2 \mathrm{eV}$, and the use of triplet spatial wavefunctions will introduce some additional error into the singlet energies. Limited electronicstructure calculations on the excited states suggest that all are dissociative, in agreement with experimental observations, and that some may lead to $\mathrm{C}-\mathrm{F}$ bond breakage.

\section{Results and discussion}

The absolute total dissociation cross section, $\sigma_{t, \text { diss }}$, for $\mathrm{C}_{2} \mathrm{~F}_{6}$ was measured at closely spaced energies from 8 to $30 \mathrm{eV}$ and at selected higher energies up to $700 \mathrm{eV}$. Some results from threshold to $700 \mathrm{eV}$ are displayed in figure 3 along with previous measurements by Winters [4]. Numerical values of all measured cross sections are listed in table 1. At higher energies, the present results are slightly smaller than those of [4], because the larger target bias in the present

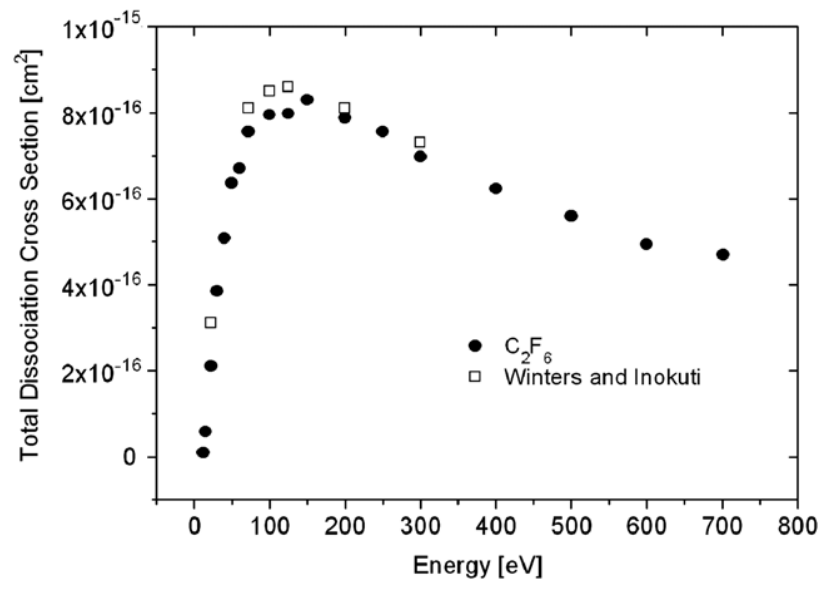

Figure 3. Comparison of previous total dissociation cross sections by Winters and Inokuti with values presented in this paper.

Table 1. Total dissociation and total ionization cross sections for $\mathrm{C}_{2} \mathrm{~F}_{6}$ in units of $10^{-16} \mathrm{~cm}^{2}$. Data in columns 2 and 4 are from this work, and data in column 3 are from [4].

\begin{tabular}{|c|c|c|c|}
\hline $\begin{array}{l}\text { Electron } \\
\text { energy } \\
{[\mathrm{eV}]}\end{array}$ & $\begin{array}{l}\text { Present } \\
\sigma_{\mathrm{t}, \text { diss }}\end{array}$ & $\begin{array}{l}\text { Winters and } \\
\text { Inokuti } \sigma_{\mathrm{t}, \text { diss }}\end{array}$ & $\begin{array}{l}\text { Present } \\
\sigma_{\mathrm{t}, \text { ion }}\end{array}$ \\
\hline 8.1 & 0 & - & - \\
\hline 8.7 & 0 & - & - \\
\hline 9.2 & 0 & - & - \\
\hline 9.7 & 0 & - & - \\
\hline 10.2 & 0.01 & - & - \\
\hline 10.7 & 0.03 & - & - \\
\hline 11 & 0 & - & - \\
\hline 12 & 0.09 & - & - \\
\hline 13 & 0.28 & - & 0.06 \\
\hline 14.1 & 0.4 & - & 0.06 \\
\hline 15 & 0.58 & - & 0.09 \\
\hline 16 & 0.74 & - & 0.12 \\
\hline 17 & 1.04 & - & 0.23 \\
\hline 18 & 1.19 & - & 0.35 \\
\hline 19 & 1.51 & - & 0.55 \\
\hline 20 & 1.73 & - & 0.77 \\
\hline 21 & 1.87 & - & 1.01 \\
\hline 22 & 2.11 & 3.1 & 1.27 \\
\hline 23 & 2.47 & - & 1.54 \\
\hline 24 & 2.64 & - & 1.8 \\
\hline 25 & 2.97 & - & 2.06 \\
\hline 26 & 3.2 & - & 2.33 \\
\hline 27 & 3.38 & - & 2.53 \\
\hline 28 & 3.5 & - & 2.75 \\
\hline 29 & 3.69 & - & \\
\hline 30 & 3.85 & - & 3.29 \\
\hline 40 & 5.08 & - & 4.91 \\
\hline 50 & 6.36 & - & 6.02 \\
\hline 60 & 6.7 & - & 6.79 \\
\hline 72 & 7.56 & 8.1 & 7.25 \\
\hline 100 & 7.95 & 8.5 & 8.38 \\
\hline 125 & 7.98 & 8.6 & 8.6 \\
\hline 150 & 8.3 & - & 8.36 \\
\hline 200 & 7.88 & 8.1 & 8.17 \\
\hline 250 & 7.56 & - & 8.06 \\
\hline 300 & 7.03 & 7.3 & 7.43 \\
\hline 400 & 6.23 & - & - \\
\hline 500 & 5.6 & - & - \\
\hline 600 & 4.94 & - & - \\
\hline 700 & 4.7 & - & - \\
\hline
\end{tabular}




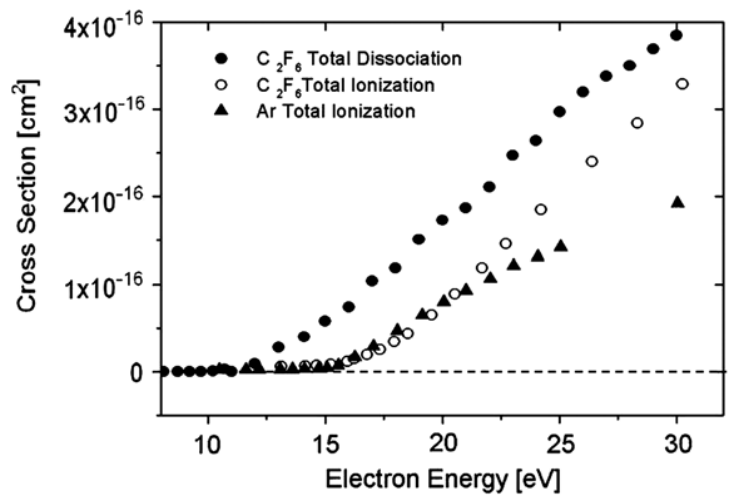

Figure 4. Absolute total dissociation cross sections for $\mathrm{C}_{2} \mathrm{~F}_{6}$ near threshold as well as total ionization cross sections for $\mathrm{C}_{2} \mathrm{~F}_{6}(\mathrm{O})$ and $\operatorname{argon}(\boldsymbol{\Delta})$, used for energy calibration.

experiments $(300 \mathrm{~V}$ as opposed to $75 \mathrm{~V}$ ) more effectively suppresses secondary electrons. The two sets of data generally agree within the quoted experimental error of $11 \%$; however, at $22 \mathrm{eV}$ the present cross section is only $70 \%$ of the previous value. This disagreement is probably due to a small error in the energy scale of the previous investigation and, in this region, the authors believe that the present measurements are more accurate. Figure 4 shows a detailed view of experimentally determined $\sigma_{\mathrm{t}, \text { diss }}$ and $\sigma_{\mathrm{t} \text {,ion }}$ of $\mathrm{C}_{2} \mathrm{~F}_{6}$ (normalized as described below) near the dissociation threshold, which is about $12 \mathrm{eV}$ for $\mathrm{C}_{2} \mathrm{~F}_{6}$, a range over which dissociation cross sections were previously unavailable. Also included in figure 4 are $\sigma_{\mathrm{t} \text {,ion }}$ for argon which were used to calibrate the energy scale as previously described.

A wider range of our experimental values for $\sigma_{t, \text { ion }}$ of $\mathrm{C}_{2} \mathrm{~F}_{6}$, normalized to the set recommended by Basner et al , [11] is shown in figure 5 . We chose to normalize to the cross sections of Basner et al in part because their technique is not susceptible to the inaccuracies due to secondary electrons or to multiply charged ions, as described above. In addition, subtraction of Basner et al's values from our measured $\sigma_{\mathrm{t} \text {, diss }}$ resulted in nonnegative values of $\sigma_{\text {neut,diss. }}$ Following normalization, our ion collection efficiency for $\mathrm{C}_{2} \mathrm{~F}_{6}$ was found to be approximately $59 \%$, in comparison with $71 \%$ for argon. Ionized fragments from dissociation of $\mathrm{C}_{2} \mathrm{~F}_{6}$ will have greater kinetic energy than the parent molecule, and the lower ion collection efficiency can be attributed to this increase in kinetic energy.

Neutral dissociation cross sections were obtained by subtraction of the $\sigma_{\mathrm{t}, \text { ion }}$ values of Basner et al [11] from the $\sigma_{\mathrm{t}, \text { diss }}$ values obtained here. The neutral dissociation cross sections so derived are compared with our calculated results (figure $6(a)$ ) and with values from other sources $[19,20]$ (figure $6(b)$ ). The agreement between the measured and calculated values is generally quite good up to the vicinity of the cross section maximum. Some of the apparent discrepancy between the calculated and measured cross sections arises from differences between the excitation thresholds assumed in the calculations and their experimental values. Though we do not do so, shifting the calculated thresholds downwards to allow for this difference would result in somewhat better agreement. At higher energies, the calculated cross section falls off too rapidly because of the omission of channels with higher thresholds than those included in the calculations.

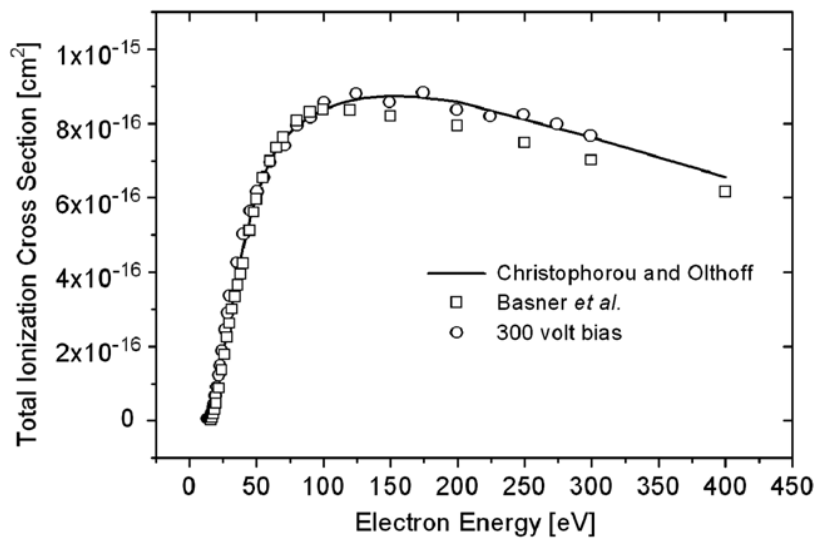

Figure 5. Total ionization cross sections for $\mathrm{C}_{2} \mathrm{~F}_{6}$ with respect to electron energy. The solid line represents values recommended by Christophorou and Olthoff, $(\square)$ are cross sections measured by Basner et $a l$ and $(O)$ represent values measured here after normalization at $100 \mathrm{eV}$. Measurements were taken with a $300 \mathrm{~V}$ bias on the electron sink.
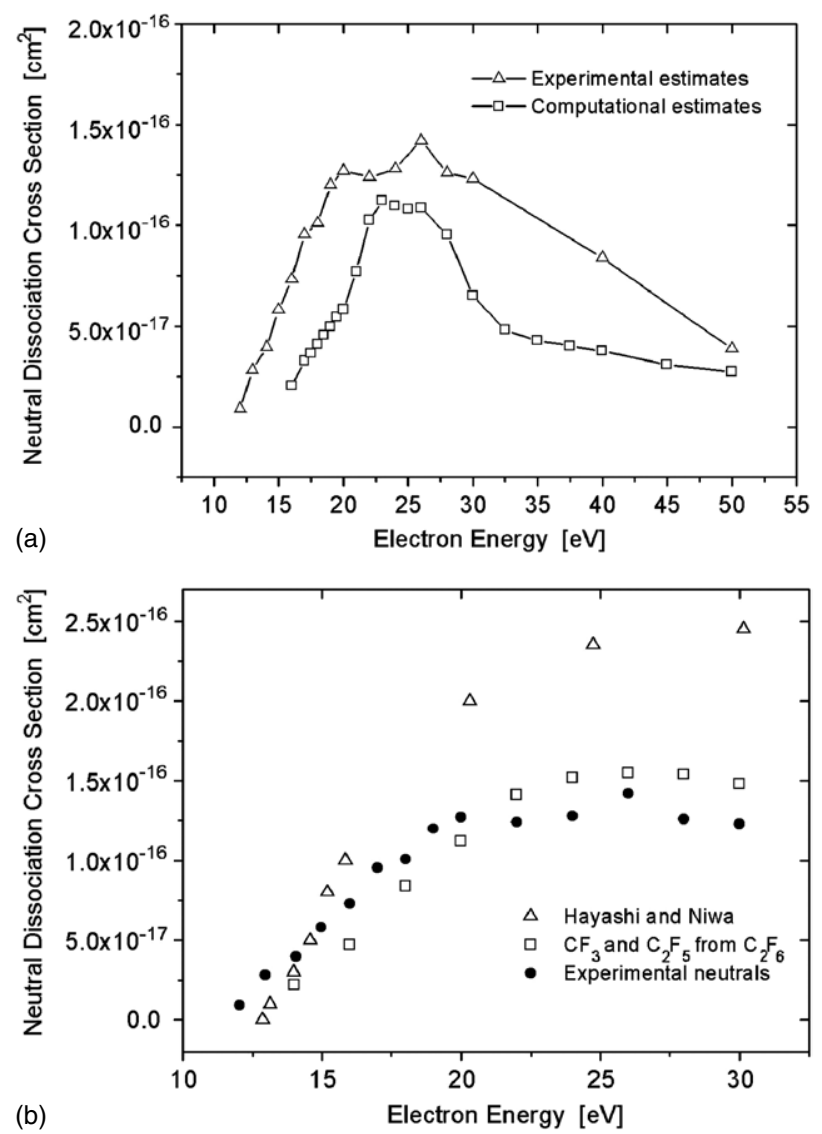

Figure 6. Neutral dissociation cross sections near threshold : (a) $(-\triangle-)$ experimental estimate; (- $\square-)$ calculated estimate; $(b)(\triangle)$ Hayashi and Niwa; ( $\square$ ) Motlagh and Moore (for partial dissociation of $\mathrm{C}_{2} \mathrm{~F}_{6}$ into $\mathrm{CF}_{3}$ and $\mathrm{C}_{2} \mathrm{~F}_{5}$ minus $\sigma_{\mathrm{p} \text {,ion }}$ for $\mathrm{CF}_{3}^{+}$from Poll and Meichsner); (๑) using $\sigma_{\mathrm{t} \text {,diss }}$ from this paper minus $\sigma_{\mathrm{t} \text {,ion }}$ measured by Basner et al.

Motlagh and Moore measured the production of $\mathrm{CF}_{3}$ and $\mathrm{C}_{2} \mathrm{~F}_{5}$ radicals from electron impact dissociation of $\mathrm{C}_{2} \mathrm{~F}_{6}$ [19]. These measurements were made by detecting the combined partial pressure of the tellurides $\mathrm{Te}\left(\mathrm{CF}_{3}\right)_{2}^{+}$and $\mathrm{FTe}\left(\mathrm{C}_{2} \mathrm{~F}_{5}\right)^{+}$ 


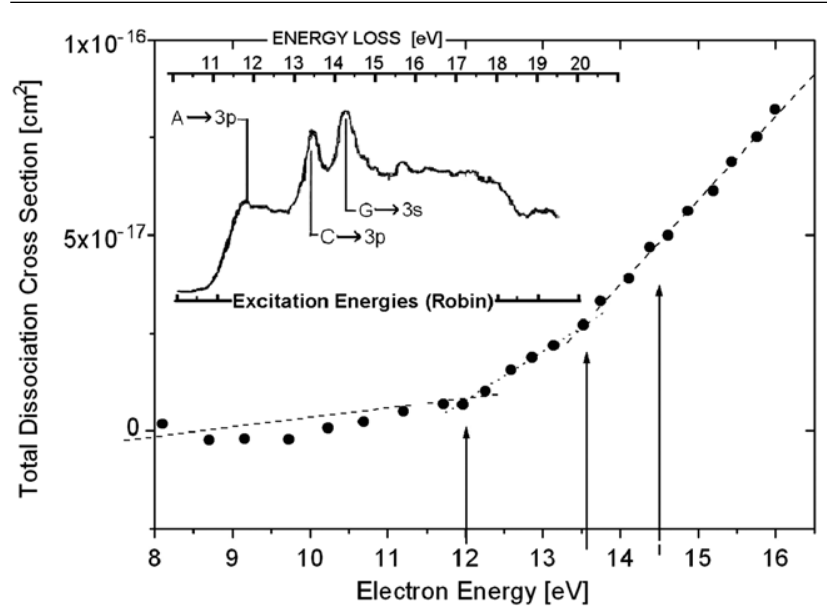

Figure 7. Total dissociation cross section in comparison with excitation energies reported by Robin (inset). Electron-impact energy-loss spectra of $\mathrm{C}_{2} \mathrm{~F}_{6}$ indicate peaks at 12.0, 13.6, $14.5 \mathrm{eV}$. Slope discontinuities can be visualized in the measured data by lines included to guide the eye.

produced by a surface reaction on a telluride mirror placed in their apparatus during the dissociation experiment. The mass spectrometer was unable to distinguish the contribution of each species individually because both compounds contribute to the $266 \mathrm{amu}$ peak that was monitored. Further, both dissociative ionization and neutral dissociation reactions contribute to this signal. Motlagh and Moore suggest that an estimate for dissociation into $\mathrm{CF}_{3}$ and $\mathrm{C}_{2} \mathrm{~F}_{5}$ in these experiments can be obtained by subtracting the partial ionization cross section, $\sigma_{\mathrm{p}, \text { ion }}$, for $\mathrm{CF}_{3}^{+}$, measured by Poll and Meichsner [21], from the sum of cross sections for dissociation into the radicals $\mathrm{CF}_{3}$ and $\mathrm{C}_{2} \mathrm{~F}_{5}$ in their experiments. The values derived from their suggested calculation lie within our estimated accuracy.

Hayashi and Niwa derived values for $\sigma_{\text {neut,diss }}$ using a Boltzmann equation method and swarm data [20]. They assumed the $\sigma_{\mathrm{t} \text { diss }}$ values of Winters and Inokuti [4] and 79\% of the total ionization cross sections of Beran and Kevan [22]. Their values are also plotted in figure $6(b)$. Our values for $\sigma_{\text {neut,diss }}$ are smaller than those proposed by Hayashi and Niwa by as much as $1.1 \times 10^{-16} \mathrm{~cm}^{2}$. This discrepancy most likely originates from the values for $\sigma_{\mathrm{t} \text {,diss }}$ and $\sigma_{\mathrm{t} \text {,ion }}$ assumed by Hayashi and Niwa. For example, at $22 \mathrm{eV}$ their assumed value for $\sigma_{\mathrm{t} \text {,diss }}$ is about $1 \times 10^{-16} \mathrm{~cm}^{2}$ smaller than the value used here. If one uses the current estimate of $\sigma_{\mathrm{t}, \text { diss }}$ at $22 \mathrm{eV}$, their value for $\sigma_{\text {neut, diss }}$ falls to $\sim 10^{-16} \mathrm{~cm}^{2}$, compared with our estimated value of $1.24 \times 10^{-16} \mathrm{~cm}^{2}$.

Finally, our values of $\sigma_{\mathrm{t} \text { diss }}$ are compared with the electron-impact energy-loss spectrum reported by Robin in figure 7 [12]. In a Rydberg state the excited electron's orbital has a much greater radius than the core orbital(s), and the core is expected to behave as an ion. This parent ion, $\mathrm{C}_{2} \mathrm{~F}_{6}^{+}$, is conspicuously absent in mass spectrometry, indicating that ionization is essentially dissociative [11]. Therefore, Rydberg excitations should result in dissociation of $\mathrm{C}_{2} \mathrm{~F}_{6}$ and should be evident in our total dissociation cross section data. Our electronic-structure studies also indicate that the low-lying excited states, at least, are indeed dissociative. The first allowed Rydberg excitation in $\mathrm{C}_{2} \mathrm{~F}_{6}$, identified by Robin as $\sigma_{\mathrm{g}} \rightarrow 3 \mathrm{p}$, occurs at $12.0 \mathrm{eV}$, and additional excitation peaks occur at 13.6 and $14.5 \mathrm{eV}$. The $12.0 \mathrm{eV}$ threshold for electronic excitation agrees with our measured threshold for dissociation at $\sim 12 \mathrm{eV}$. In addition, we see an apparent change in the slope of the total dissociation cross section at $\sim 13.5 \mathrm{eV}$ which corresponds with the second transition described by Robin. Robin attributes the third peak in the spectrum, at $14.5 \mathrm{eV}$, to the vertical ionization potential. Currently, Christophorou and Olthoff [23] suggest an ionization potential of $15.9 \mathrm{eV}$, close to our measured threshold for ionization of $\sim 16.6 \mathrm{eV}$. Recall that our estimate for electron energy scale resolution is $\pm 0.2 \mathrm{eV}$. We conclude that our data for $\sigma_{\mathrm{t} \text {,diss }}$ are consistent with this independent measurement of the electron energy loss spectrum. Non-zero values of the dissociation cross section below $12 \mathrm{eV}$ are attributed either to an artefact or to dissociative attachment reactions.

\section{Summary and conclusions}

We have reported on the results of measurements and calculations of cross sections for electron impact dissociation of $\mathrm{C}_{2} \mathrm{~F}_{6}$ into neutrals as a function of electron energy, emphasizing the near-threshold region. Our estimates of these cross sections in this technologically important region are smaller than the selected values previously published using the same apparatus and method, and the difference is attributed to more effective control of secondary electrons in the apparatus. At $22 \mathrm{eV}$ the value of $\sigma_{\mathrm{t} \text {, diss }}$ is $2.1 \times 10^{-16} \mathrm{~cm}^{2}$ in comparison with the previous value of $3.1 \times 10^{-16} \mathrm{~cm}^{2}$.

Extrapolation of the total dissociation data yields a threshold of $12 \mathrm{eV}$ for dissociation. This threshold closely corresponds to the first allowed Rydberg excitation seen in the measured electron-impact energy-loss spectrum for $\mathrm{C}_{2} \mathrm{~F}_{6}$. An additional Rydberg excitation appears as a change in the slope in the total dissociation cross section data.

Values for $\sigma_{\text {neut,diss }}$ derived from our measurements plateau at $\sim 20 \mathrm{eV}$ with a value of $1.3 \times 10^{-16} \mathrm{~cm}^{2}$, in good agreement with the maximum $\left(1.1 \times 10^{-16}\right.$ at $\left.23 \mathrm{eV}\right)$ obtained from our $a b$ initio calculations. Discrepancies between these values and those of Hayashi and Niwa may be rooted in differences between the values they used for $\sigma_{\mathrm{t} \text {,diss }}$ and $\sigma_{\mathrm{t} \text {,ion }}$ and those proposed here. The estimated cross sections for dissociation into $\mathrm{CF}_{3}$ and $\mathrm{C}_{2} \mathrm{~F}_{5}$ reported by Motlagh and Moore are within the error limits of our estimates of total dissociation cross section into all neutral species. Thus, recent experimental and theoretical results from several independent investigations now give a unified and consistent picture of dissociation of $\mathrm{C}_{2} \mathrm{~F}_{6}$.

\section{Acknowledgments}

The authors acknowledge the IBM Almaden Research Center for donating much of the experimental apparatus. This research was funded in part by the NSF-SRC ERC for Environmentally Benign Semiconductor Manufacturing. Work by CW and VM was funded by Sematech Inc. and by the Department of Energy, Office of Basic Energy Sciences. The authors gratefully acknowledge Professor J H Moore for discussions and unpublished data. Additionally, assistance from Bing Ji of Air Products is greatly appreciated. 


\section{References}

[1] Christophorou L G and Olthoff J K 2002 Appl. Surf. Sci. 192309

[2] Tonnis E J, Graves D B, Vartanian V H, Beu L, Lii T and Jewett R 2000 J. Vac. Sci. Technol. A 18 393-400

[3] Christophorou L G and Olthoff J K 1998 J. Phys. Chem. Ref. Data 271

[4] Winters H F and Inokuti M 1982 Phys. Rev. A 251420

[5] Winters H F 1966 J. Chem. Phys. 441472

[6] Winters H F, Horne D E and Donaldson E E 1964 J. Chem. Phys. 412766

[7] Winters H F 1975 J. Chem. Phys. 633462

[8] Winters H F 1979 Chem. Phys. 36353

[9] Mi L and Bonham R A 1998 J. Chem. Phys. 1081910

[10] Takatsuka K and McKoy V 1981 Phys. Rev. A 242473

Takatsuka K and McKoy V 1984 Phys. Rev. A 301734

[11] Basner R, Schmidt M, Denisov E, Lopata P, Becker K and Deutsch H 2002 Int. J. Mass Spectrom. 214365
[12] Robin M B 1974 Higher Excited States of Polyatomic Molecules (New York: Academic) vol 1 p 188

[13] Rapp D and Englander-Golden P 1965 J. Chem. Phys. 431464

[14] Taylor J R 1997 An Introduction to Error Analysis: The Study of Uncertainties in Physical Measurements (Sausalito, CA: University Science Books) p 56

[15] Winstead C and McKoy V 2000 Comput. Phys. Commun. 128386

[16] Lima M A P, Brescansin L M, da Silva A J R, Winstead C and McKoy V 1990 Phys. Rev. A 41327

[17] Krishnan R, Binkley J S, Seeger R and Pople J A 1980 J. Chem. Phys. 72650

[18] Hunt W J and Goddard W A 1969 Chem. Phys. Lett. 3414

[19] Motlagh S and Moore J 1998 J. Chem. Phys. 109432

[20] Hayashi M and Niwa A 1987 Gaseous Dielectrics V ed L G Christophorou and D W Bouldin (New York: Pergamon) p 27

[21] Poll H U and Meichsner J 1987 Contrib. Plasma Phys. 27359

[22] Beran J A and Kevan L 1969 J. Phys. Chem. 733866

[23] Christophorou L G and Olthoff J K 1999 J. Phys. Chem. Ref. Data 28967 